\title{
Evaluation and identification of poly-microbial biofilms on natural green Gordal table olives
}

Antonio Benítez-Cabello · Verónica Romero-Gil · Francisco Rodríguez-Gómez · Antonio Garrido-Fernández • Rufino Jiménez-Díaz · Francisco Noé Arroyo-López*

Food Biotechnology Department. Instituto de la Grasa (Agencia Estatal Consejo Superior de Investigaciones Científicas, CSIC). University Pablo de Olavide, Building 46, Ctra. Utrera, km 1, 41013-Seville, Spain

Running title: Biofilm formation on natural green olives

Corresponding author: Francisco Noé Arroyo-López, Ph.D. Tel: 0034954611550 ext 142. e-mail: fnarroyo@cica.es 


\section{Abstract}

2 This work examines the formation of poly-microbial communities adhered to the

3 epidermis of natural green Gordal olives and the application of different methodologies for

4 recovery and counting of the microorganisms embed in olive biofilms. The fermentation

5 process was physicochemical and microbiologically monitored for $90 \mathrm{~d}$, at which,

6 formation of true biofilms on the skin of fermented fruits was confirmed by scanning

7 electron microscopy. Then, samples of olives were taken and treated with sonication,

8 enzymes, mechanic homogenization with stomacher and ultrasonic bath for biofilm

9 disaggregation. The use of the stomacher for 1 min was the most effective treatment to 10 release the lactic acid bacteria $\left(6.6 \log _{10} \mathrm{cfu} \cdot \mathrm{g}^{-1}\right)$, whereas sonication for 5 min was the 11 most efficient method for quantification of yeasts (up to $3.5 \log _{10} \mathrm{cfu} \cdot \mathrm{g}^{-1}$ ). Molecular 12 identification of isolates obtained from natural Gordal olive biofilms revealed that 13 Lactobacillus pentosus was the only species found among lactic acid bacteria, while Pichia membranifaciens was the dominant yeast species, with higher counts obtained for the bacteria.

Keywords: Biofilm analysis · natural fermented olives • lactic acid bacteria $\cdot$ table olives • yeasts 


\section{Introduction}

Spain generates almost a quarter of the worldwide table olive production, which nowadays exceeds 2.5 million tons per year (IOC 2013). Among the diverse processing methods, alkali-treated green olives (Spanish style), ripe olives by alkaline oxidation (Californian style) and directly brined olives (natural olives) are the most common (Garrido-Fernández et al. 1997). However, only directly brined olives are produced without alkaline treatment. Thereby, the fresh fruits, after a wash to remove dirty and impurities, are placed in a $7-10 \% \mathrm{NaCl}$ solution where the addition of different organic acids (citric, acetic or lactic acid) to decrease the initial $\mathrm{pH}$ is a common practice. In this way, the olive sweetening is achieved by diffusion of the bitter glucoside oleuropein from fruits into the cover brines, where it is finally hydrolysed.

Lactic acid bacteria (LAB) are the most important microorganisms responsible for the fermentation of $\mathrm{NaOH}$ treated table olives and other fermented vegetables (Hurtado et al. 2012; Pérez-Díaz et al. 2013). By sugars consumption and subsequent production of lactic acid and other antimicrobial metabolites, the LAB population contributes to the safe preservation of olives by formation of lactic acid, reduction of $\mathrm{pH}$ and production of bacteriocins. In directly brined natural olives, both LAB and yeasts may usually coexist along the entire process although, sometimes, yeasts can play a more relevant role in the fermentation due to partial inhibition of LAB by the presence of phenolic compounds (Aponte et al. 2010; Balatsouras 1990; Brenes 2004; Garrido-Fernández et al. 1997; Sánchez et al. 2000; Tassou et al. 2002). Thereby, regardless of olive processing, both groups of microorganisms determine the quality, safety and flavour of the final products. 
For many years, the microbiological study of table olive fermentations has been

exclusively focused on the isolation, identification and characterization of microorganisms present in brines. However, recent studies carried out with table olives have shown the presence of polymicrobial communities adhered to both biotic (olive skin) and abiotic (glass slides) surfaces during the fermentation process (Arroyo-López et al. 2012a; Domínguez-Manzano et al. 2012; Nychas et al. 2002). As observed by scanning electron microscopy (SEM), these polymicrobial communities consisted of different yeast and bacteria species embedded in a matrix which keeps them in close proximity. Detachment of microorganisms from olive skin to determine the number of cells and further molecular identification using a protocol consisting of an enzymatic method and RAPD analysis, revealed the presence of Pichia galeiformis, Candida sorbosa and Geotrichum candidum for the yeast species, and Lactobacillus pentosus for the LAB population (Arroyo-López et al. 2012a; Domínguez-Manzano et al. 2012). After detachment, both yeasts and bacteria species yielded high population levels $\left(>7 \log _{10} \mathrm{cfu} \cdot \mathrm{g}^{-1}\right)$, thus showing that the olives could be a good carrier of microorganisms. However, this methodology implies a wide variation in the number of microbial cells recovered, which also depends on the group of microorganisms. In fact, the cocktail of enzyme detaches completely the LAB population after $6 \mathrm{~h}$ of incubation whereas the release of yeasts requires up to $16 \mathrm{~h}$ treatment (personal communication). Greek researches have also evaluated mechanic disaggregation with stomacher for detachment of Lactobacillus pentosus and Pichia membranifaciens species from ripe black (darkened by oxidation) olives with good results, obtaining $>7$ $\log _{10} \mathrm{cfu} \cdot \mathrm{g}^{-1}$ (Grounta and Panagou 2014). However, comparison of results is difficult because the use of different methodologies. Thus, bearing in mind the transcendence of further studies on olive biofilms, the standardization of a rapid and accurate procedure to recover microbes from these fruits is needed. 
In this work, we study the fermentation process and the formation of true biofilms on natural green Gordal table olives. For the quantification of the microbial populations on olives, several methods for detachment, recovery and counting of microorganisms attached to fruits have been assessed. Furthermore, the biodiversity of the most important LAB and yeast species present until now unexplored biofilms formed in this type of table olive preparation was investigated by molecular methods.

\section{Material and methods}

Olive fermentations

Olive fruits from Gordal variety were obtained during the 2013/2014 season at the green ripening stage from the olive processing plant Ntra. Sra. de las Virtudes S.C.A. (La Puebla de Cazalla, Seville, Spain), and transported to our laboratory where they were classified by size, washed and directly brined in polyethylene fermentation vessels. The process was achieved as industry, by immersing $20 \mathrm{~kg}$ of fruits into $13 \mathrm{l}$ of brine (10\% $\mathrm{NaCl}, 0.5 \%$ acetic acid and $0.1 \%$ citric acid). The fermentation was let to evolve spontaneously. The study was carried out in two independent fermentation vessels and monitored during $90 \mathrm{~d}$.

\section{Analysis of the fermentation brines}

Physicochemical control of the fermentation was achieved through periodical analyses of brine $(0,10,20,40,60$ and $90 \mathrm{~d})$ for determination of $\mathrm{pH}, \mathrm{NaCl}$ concentration $\left(\%, \mathrm{wt} \cdot \mathrm{vol}^{-1}\right)$, titratable acidity, expressed as g lactic acid per $100 \mathrm{ml}$ of brine, and combined acidity (undissociated organic salts, expressed as Eq·l-1) (Garrido-Fernández et al. 1997). 
To study the evolution of the different microbial populations, brine samples were taken at different times throughout fermentation $(0,3,6,10,20,30,60,90 \mathrm{~d})$ and diluted, if necessary, in a sterile saline solution $(0.9 \% \mathrm{NaCl})$. Then, they were plated using a Spiral System (model dwScientific, Don Whitley Scientific Limited, England) on appropriate media. Enterobacteriaceae were counted on Crystal Violet Neutral-Red Bile Glucose (VRBD) agar (Merck, Darmstadt, Germany), LAB were proliferated on de Man, Rogosa and Sharpe (MRS) agar (Oxoid, Basingstoke, Hampshire, England) supplemented with $0.02 \%$ sodium azide (Sigma, St. Luis, USA), and yeasts were grown on yeast-malt-peptone-glucose medium (YM) agar (Difco, Becton and Dickinson Company, Sparks, MD, USA) supplemented with oxytetracycline and gentamicin sulphate $(0.005 \%)$ as selective agents. The plates were incubated at $30^{\circ} \mathrm{C}$ for $48-72 \mathrm{~h}$ and counted using a CounterMat v.3.10 (IUL, Barcelona, Spain) image analysis system. Brine counts were expressed as $\log _{10} \mathrm{cfu} \cdot \mathrm{ml}^{-1}$.

The plot of the $\log _{10} \mathrm{cfu} \cdot \mathrm{ml}^{-1}$ versus time for microorganisms produced a sigmoidshape curve that was fitted using the reparameterized Gompertz equation proposed by Zwietering et al. (1990), which has the following expression:

$$
y=N_{\max } * \exp \left(-\exp \left\{\left(\mu_{\max }{ }^{*} \mathrm{e}^{*}(\lambda-x)\right) / N_{\max }+1\right\}\right)
$$

where $y$ is the microbial concentration $\left(\log _{10} \mathrm{cfu} \cdot \mathrm{ml}^{-1}\right)$ at time $\mathrm{t}, x$ is the time (days), $N_{\max }$ is the maximum population reached $\left(\log _{10} \mathrm{cfu} \cdot \mathrm{ml}^{-1}\right), \mu_{\max }$ is the maximum growth rate $\left(\mathrm{d}^{-1}\right)$ and $\lambda$ is the lag phase (d). These parameters were obtained by a nonlinear regression procedure, minimizing the sum of squares of the difference between the experimental data and the fitted model, i.e. loss function (observed - predicted). This task was accomplished using the nonlinear module of the Statistica 7.1 software package (StatSoft Inc, Tulsa, OK, USA) and its Quasi-Newton option. Fit adequacy was checked by the proportion of total variance explained by the model $\left(R^{2}\right)$. 
In situ observation of olive epidermis was confirmed by using SEM techniques. For this purpose, olives were treated following the methodology described by Krouwilleypitski et al. (2009) with slight modifications. First, fruits were rinsed twice for $15 \mathrm{~min}$ in a PBS buffer solution $\left(8.0 \mathrm{~g} \cdot \mathrm{l}^{-1} \mathrm{NaCl}, 0.2 \mathrm{~g} \cdot \mathrm{l}^{-1} \mathrm{KCl}\right.$, $1.44 \mathrm{~g} \cdot \mathrm{l}^{-1} \mathrm{Na}_{2} \mathrm{HPO}_{4}, 0.24 \mathrm{~g} \cdot \mathrm{l}^{-1} \mathrm{KH}_{2} \mathrm{PO}_{4}, \mathrm{pH} 7.4$ adjusted with $\mathrm{HCl} 1 \mathrm{M}$ ) for removing nonadhering cells, and then fixed in $2.5 \%$ glutaraldehyde (Sigma-Aldrich, St. Louis, USA) in PBS for $2.5 \mathrm{~h}$. Later, the olives were dehydrated through a graded ethanol series (50\%, 70\%, 80\%, 90\%, 95\% and 100\%, 5 min in each one). Finally, fruits were treated for 20 min in 2-methyl-2-propanol. For SEM observation, $2 \mathrm{~mm}^{2}$ slices of the skin of olives were taken and placed on glass slides and coated with gold in a Scancoat Six SEM sputter coater (Edwards, Crawley, England). Pictures were taken with a JEOL JSM- 6460LV SEM model (JEOL USA, Inc., Peabody, MA) in the Technology and Innovation Research Center at University of Seville (CITIUS, Seville, Spain).

Assessment of the efficacy of different methodologies for the detachment/recovery of microorganisms from biofilms and fruits

All methods described below were applied to 2 olives removed under sterile conditions from the fermentation vessels at the end of the fermentation process (90 d), except the ultrasonic bath which used 5 fruits. For removing microbial non-adhered cells, fruits were previously washed for $30 \mathrm{~min}$ in sterile distilled water, weighed (to further refer plate counts of microorganisms as $\log _{10} \mathrm{cfu} \cdot \mathrm{g}^{-1}$ ) and spread (after application of different treatments) onto the different culture media specific for Enterobacteriaceae, yeasts and LAB. Values (means and standard deviations) were obtained from 6 measurements per level $(n=6)$, with three technical replicates per independent duplicate. 
The protocol developed by Böckelmann et al. (2003) was slight adapted to the specific characteristics of table olives. Three different types of enzymes (lipase, $\beta$ galactosidase and $\alpha$-glucosidase) were purchased (Sigma-Aldrich, St. Louis, USA) and mixed in the laboratory to obtain an enzymatic cocktail with the following concentrations: lipase $\left(0.74 \mathrm{mg} \cdot \mathrm{ml}^{-1}\right), \beta$-galactosidase $\left(0.64 \mathrm{mg} \cdot \mathrm{l}^{-1}\right)$, and $\alpha$-glucosidase $\left(1.05 \mu \mathrm{L}^{-\mathrm{ml}^{-1}}\right) . \alpha-$ glucosidase and $\beta$-galactosidase were chosen for the cleavage of the $\alpha$-D-glucoside residues and $\beta$-galactosidic bonds of exopolysaccharides, respectively, while lipase was added to the enzyme mixture as lipids represent a considerable part of this component from biofilms (Böckelmann et al. 2003). It was used at full (standard), half $(1 / 2)$, double $(\times 2)$ and four (x4) times concentrations taking as references previous works carried out in table olives (Arroyo-López et al. 2012a; Domínguez-Manzano et al. 2012). The fruits were incubated at $30{ }^{\circ} \mathrm{C}$ for $1 \mathrm{~h}$ in $50 \mathrm{ml}$ of PBS buffer containing the different enzyme preparations. The resultant suspension was centrifuged at $9,000 \times g$ for $10 \mathrm{~min}$ at $4^{\circ} \mathrm{o}$, the pellet was resuspended in $2 \mathrm{ml}$ of PBS buffer and finally spread.

\section{Sonication method}

In this case, fruits were immersed into $50 \mathrm{ml}$ of a sterile saline solution $(0.9 \% \mathrm{NaCl})$, and then sonicated using an ultrasonic liquid processor model Microson ${ }^{\mathrm{Tm}}$ XL 2000 (QSonica LLC., Newtown, CT, USA) which works at a wave frequency of $22.5 \mathrm{kHz}$. The processing tip of the sonicator was dipped $1 \mathrm{~cm}$ in the liquid. The olives were sonicated for $0.08,0.016,0.5,1,2,5,10,15,20$ and $30 \mathrm{~min}$ at an ultrasound power of $6 \mathrm{~W}(50 \%$ of the total intensity). Suspension of the appropriate dilutions were spread plated.

\section{Stomacher method}


Fruits were pitted, weighed and immediately transferred into a stomacher bag containing $75 \mathrm{ml}$ of a sterile saline solution $(0.9 \% \mathrm{NaCl})$. Then, pulp was homogenized for 1, 5, 10, 15 and $20 \mathrm{~min}$ at maximum speed $(300 \mathrm{rpm})$ in a stomacher model Seward 400 (Seward Medical, Ltd., West Sussex, England). Suspension of the appropriate dilutions were then spread plated.

\section{Ultrasonic bath method}

Fruits were immersed into $35 \mathrm{ml}$ of a sterile saline solution $(0.9 \% \mathrm{NaCl})$ and treated with an ultrasound bath model Ultrasons 3000513 (J.P. Selecta, S.A., Barcelona, Spain), which works at a power of $360 \mathrm{~W}$. The olives were treated for 1, 5, 10, 15, 20 and $40 \mathrm{~min}$. Samples of the resulting suspensions were taken, diluted in saline solution if needed, and then spread plated. During the entire process, the water in the bath was kept constant at 30 ${ }^{\circ} \mathrm{C}$ by adding ice.

\section{Molecular characterization and identification of microorganisms}

For characterization of yeast isolates, a RAPD-PCR analysis with M13 primer was followed according to the protocol described by Tofalo et al. (2009), while in the case of lactobacilli, a rep-PCR analysis was performed using GTG 5 primer (Gevers et al. 2001). PCR products were electrophoresed in a $2 \%$ agarose gel, stained with ethidium bromide (20 min) and visualized under ultraviolet light. The resulting fingerprints were digitally captured and analysed with the Bio-Numerics 6.6 software package (Applied Maths, Kortrijk, Belgium). The similarity among digitalized profiles was calculated using the Pearson product-moment correlation coefficient. The dendrogram was generated by means of the Unweighted Pair Group Method using the Arithmetic Average (UPGMA) clustering algorithm. The reproducibility and sensitivity of the method was previously evaluated 
using, as internal control, 7 LAB and 8 yeast strains belonging to species Lactobacillus pentosus, Lactobacillus plantarum, Lactobacillus paraplantarum, Saccharomyces cerevisiae, Wickerhamomyces anomalus, Candida boidinii and Pichia galeiformis obtained from the Table Olives Microorganisms Collection from Instituto de la Grasa (CSIC, Spain) (data not shown). Reproducibility of the technique, in the worst case, was determined in $85.1 \%$ and $80.5 \%$ for LAB and yeasts, respectively.

Then, molecular identification of representative genotypes was performed using multiplex PCR of recA gene (Torriani et al., 2001) and RFLP analysis of $d n a K$ gene (Huang et al. 2010) in the case of LAB, or RFLP analysis of 5.8S ITS region (Esteve-Zarzoso et al. 1999) in the case of yeasts. The yeast profiles generated were then compared with existing databases (www.yeast-id.org, University of Valencia and CSIC, Spain).

\section{Statistical analysis}

An analysis of variance was performed by means of the one-way ANOVA module of Statistica 7.1 software to check for significant differences among different levels and microbial recovery methods. For this purpose, a post-hoc comparison was applied by means of the Scheffé test.

\section{Results and discussion}

\section{Evolution and control of fermentation}

Titratable acidity and $\mathrm{pH}$ are critical parameters to monitor completion of a safe olive fermentation and control the growth of spoilage and pathogenic microorganisms during fermentation (Garrido-Fernández et al. 1997; Perricore et al. 2010). In this experiment cover brine $\mathrm{pH}$ increased rapidly from an initial value of 2.5 to 3.5 after olive 
brining (Figure 1a), due to the diffusion of the organic acids into the flesh. The equilibrium between the olive flesh and cover brine was reached on day 9 , after which the $\mathrm{pH}$ value oscillated around 3.5 units until the end of the fermentation. On the contrary, titratable acidity decreased during the first 18 days from 0.95 to $0.80 \mathrm{~g}$ lactic per $100 \mathrm{ml}$ due to, as in the case of $\mathrm{pH}$, the absorption of organic acids by the pulp. However, a progressive increase was observed after the $30^{\text {th }}$ day, possibly due to the production of lactic acid by the LAB population, which reached a final value of approximately $1.1 \mathrm{~g}$ lactic per $100 \mathrm{ml}$ brine in the processed product (Figure 1b). Combined acidity increased throughout the fermentation from initial 0.000 to final $0.035 \mathrm{Eq} \cdot \mathrm{l}^{-1}$, while salt concentration decreased from the initial 6.0 to a final $4.5 \% \mathrm{NaCl}$, showing the major drop during the first 10 days (data not shown). These changes in $\mathrm{pH}$ and salt, together with combined and titratable acidities obtained, are typical of directly brined table olive fermentations (Garrido-Fernández et al. 1997). Furthermore, the $\mathrm{pH}$ value far below the limit established for green natural olives $(<4.3)$ in the Table Olive Standard, and the titratable acidity value above $1.0 \mathrm{~g}$ lactic per $100 \mathrm{ml}$ brine are important aspects to ensure a safe product (Garrido-Fernández et al. 1997; IOC 2004). Hence, these natural green Gordal olives followed an adequate fermentation process from the physicochemical point of view.

Regarding evolution of microbial populations in brines, Enterobacteriaceae were not detected along the $90 \mathrm{~d}$ of the fermentation process. Low $\mathrm{pH}$ levels have showed to exert a considerable inhibitory effect on this microbial group (Garrido-Fernández et al. 1997). On the contrary, LAB and yeast populations in brine showed the typical growth for this type of processes. Their evolutions could be well fitted with the reparameterized Gompertz equation for growth (Zwietering et al. 1990), with a $\mathrm{R}^{2}$ (quality of the adjustment) of 0.987 for LAB and 0.865 for yeasts (Figure 2). The fitted parameters obtained for LAB population (Figure $2 \mathrm{a}$ ) showed a lag phase $(\lambda)$ of $3.649 \pm 0.778 \mathrm{~d}$, a maximum growth rate $\left(\mu_{\max }\right)$ of 
$0.669 \pm 0.106\left(\mathrm{~d}^{-1}\right)$ and a maximum population size $\left(N_{\max }\right)$ of $6.727 \pm 0.239\left(\log _{10} \mathrm{cfu} \cdot \mathrm{ml}^{-1}\right)$. In the case of yeasts (Figure $2 \mathrm{~b}$ ), the values obtained were: $\lambda=0.227 \pm 3.483 \mathrm{~d}, \mu_{\max }=$ $0.228 \pm 0.079 \mathrm{~d}^{-1}$, and $N_{\max }=5.066 \pm 0.687 \log _{10} \mathrm{cfu} \cdot \mathrm{ml}^{-1}$. Therefore, the process was clearly dominated by $\mathrm{LAB}$, with higher growth rate than yeast ( 0.669 vs $\left.0.228 \mathrm{~d}^{-1}\right)$ and also maximum population levels (6.73 vs $5.1 \log _{10} \mathrm{cfu} \cdot \mathrm{ml}^{-1}$ ) in brines, which were obtained approximately at the $30^{\text {th }}$ day of fermentation (Figure 2) and remained stable until the end of the process. The counts and behaviour obtained for both microbial groups throughout the fermentation process can also be considered suitable for this type of table olive elaboration (Arroyo-López et al. 2012b; Nychas et al. 2002).

SEM

Nychas et al. (2002) reported for the first time using SEM techniques the presence of both LAB and yeast populations colonizing the epidermis of fermented Greek black olives. However, these authors did not report the presence of a matrix surrounding microorganisms (true biofilms). Years later, the formation of true mixed biofilms (with exopolyssacharide matrix) between LAB and yeasts during Spanish-style green table olive fermentations was reported for different types of olive varieties by Arroyo-López et al. (2012a) and Domínguez-Manzano et al. (2012). Recently, Grounta and Panagou (2014) also have showed by SEM the formation of biofilms on Greek black oxidized olives. In this work, we describe for the first time the formation of microbial biofilms on the epidermis of Gordal fruits processed as green directly brined "natural" olives.

At the end of the fermentation, both LAB and yeasts appear to be strongly adhered to the epidermis of olives and embedded in a matrix, which is a clear evidence of the presence of true biofilms in this type of table olive elaboration (Figure 3). SEM pictures also 
show some microbial cells apparently ready for leaving the biofilms, or just trying to find physical space to form a thicker layer.

\section{Comparison of different methods for quantification and recovery of biofilms}

Usually, once the biofilm has been formed, the microorganisms are strongly adhered to the epidermis of the olives and are not released with a simple washing procedure (Arroyo-López et al. 2012a; Domínguez-Manzano et al. 2012). Furthermore, the efficiency of the procedures for the biofilm recovery has been scarcely tested. Olives from this experiment have been used to compare different procedures for detachment and quantification of microorganisms forming biofilms. The efficacy of each treatment was measured by statistical analysis of the microorganism mean counts released after its application.

\section{Sonication method}

When a biofilm is sonicated, microorganisms are detached by a mechanism named cavitation. This term refers to the generation, growth and collapse of microbubbles in the sonicated liquid. The changes in pressure can lead to the biofilm disaggregation (Piyasena et al. 2003). In addition of temperature and viscosity of the liquid, frequency and amplitude of the ultrasonic waves influence the degree of cavitation and therefore the effectiveness of the treatment (Mason et al. 1996; Sala 1995). A previous work has reported bactericide and bacteriostatic effects by gradually increasing time and intensity of sonication (Tsukamoto et al. 2004). In this work, sonication was fixed at medium intensity (6W), varying sonication times to determine the more effective time to disaggregate the biofilm and removing the microorganisms without producing lysis or cell inactivation. 

populations from the Gordal olive biofilms showed that the LAB counts released from the

biofilm were higher than those of yeasts, and that both group of microorganisms increased their detachment as the time of sonication increased up to $15 \mathrm{~min}$ (Figure 4). Thereby, there was a significant difference in LAB population between the lowest time of sonication $(0.083 \mathrm{~min})$ and the longer treatment $(30 \mathrm{~min})$, which released $\sim 4 \log _{10} \mathrm{cfu} \cdot \mathrm{g}^{-1}$ and $\sim 6$ $\log _{10} \mathrm{cfu} \cdot \mathrm{g}^{-1}$, respectively. Sonication for periods above $5 \mathrm{~min}$ led to similar counts (Table 1). On the contrary, there were no significant differences among the yeast populations from the diverse treatment levels (period of times), and the counts ranged from $\sim 2 \log _{10} \mathrm{cfu} \cdot \mathrm{g}^{-1}$ $(0.166 \mathrm{~min})$ to $\sim 4 \log _{10} \mathrm{cfu} \cdot \mathrm{g}^{-1}(10 \mathrm{~min})$.

\section{Enzymatic method}

Detachment of biofilms in table olives by using a cocktail of enzymes has been previously reported in the literature (Arroyo-López et al., 2012a; Domínguez-Manzano et al. 2012). Usually, an incubation time of $12 \mathrm{~h}$ is applied. However, in this work we have used lower incubation time $(1 \mathrm{~h})$ to avoid exceeding the generation time of LAB and yeasts, which according to the literature, in optimal conditions, is approximately $1.1 \mathrm{~h}$ for many species of LAB, and $2 \mathrm{~h}$ for the growth-faster yeast species (Brizuela et al. 2001; Nagpal and Kaur 2011; Willey et al. 2011). In this way, duplication of the microorganisms that are released is prevented and time is reduced. Böckelmann et al. (2003) used an incubation time of 90 min for detachment of biofilms from soils using the same cocktail of enzymes. No bacterial growth was observed during treatment for this period of time.

After application of the enzymatic method, LAB population levels obtained from biofilms were considerably higher (approx. $5 \log _{10} \mathrm{cfu} \cdot \mathrm{g}^{-1}$ ) than yeasts (about $1.5 \log _{10}$ $\left.\mathrm{cfu} \cdot \mathrm{g}^{-1}\right)$, with no statistical significant differences between different levels of the enzyme 
cocktail within the same microbial group (see Table 1 and Figure 4). Therefore, according to the data presented in this study, the enzyme cocktail used in the literature could be reduced to a half concentration without a loss of effectiveness in the detachment of biofilms from the olives (Arroyo-López et al. 2012a; Dominguez-Manzano et al. 2012). Due to the heterogeneity of the extracellular polysaccharides, a mixture of enzymes activities is usually necessary for destabilization of biofilms (Arroyo-López et al. 2012a; DominguezManzano et al. 2012). These enzymes have targets for the lipids, $\alpha$-D-glucoside residues and $\beta$-galactosidic bonds present in the exopolysaccharide matrix (Böckelmann et al. 2003).

\section{Stomacher method}

Release of microorganisms from biofilms using a stomacher apparatus is basically a physical method where the entire structure of the olives, and consequently the biofilms, are disaggregated by using paddles to homogenize the food sample immersed into a liquid medium. This method is widely used in the literature to count microorganisms in solid foods in which 1-2 min of application is currently used (Grounta and Panagou 2014; Medina et al. 2007).

After application of stomacher for different periods of time, the population levels of LAB obtained (from 6.5 to $7.0 \log _{10} \mathrm{cfu} \cdot \mathrm{g}^{-1}$ ) were much higher than those of yeasts (in many cases lower than $1 \log _{10} \mathrm{cfu} \cdot \mathrm{g}^{-1}$ ) with no statistical significant differences between times of application within the same microbial group (Table 1, Figure 4). In table olives, Grounta and Panagou (2014) used a stomacher time of 2 min to recover microorganisms present in fruits, obtaining a maximum recovery of $7 \log _{10} \mathrm{cfu} \cdot \mathrm{g}^{-1}$ for bacteria and $5 \log _{10} \mathrm{cfu} \cdot \mathrm{g}^{-1}$ for yeasts. 


\section{Ultrasonic bath method}

Ultrasonic baths are commonly used for the sterilization of laboratory and medical material (Raffin et al. 2008). By immersing the samples into a liquid medium, the ultrasonic wave is applied in different directions setting a specific frequency. The immersion of the naturally fermented olives for diverse periods of time in an ultrasound bath working at 50 $\mathrm{Hz}$, showed that LAB population levels obtained $\left(\sim 5 \log _{10} \mathrm{cfu} \cdot \mathrm{g}^{-1}\right)$ were higher than those of yeasts (frequently lower than $1 \log _{10} \mathrm{cfu} \cdot \mathrm{g}^{-1}$ ), with no statistical significant differences between application times within the same microbial group (Table 1, Figure 4).

\section{Statistical comparison between methods}

Table 1 shows the average counts obtained for the different methods and levels assayed. As can been deduced, there were not statistical significant differences within the same detachment methodology among the different levels, except for sonication in the release of $\mathrm{LAB}$.

As a summary, Table 2 shows the statistical comparison (Scheffé test) among the greater LAB and yeast counts obtained within methodologies. The statistical analysis shows that the best method (highest counts) for recovery of LAB was stomacher applied for $1 \mathrm{~min}\left(6.6 \log _{10} \mathrm{cfu} \cdot \mathrm{g}^{-1}\right)$ whereas sonication for $5 \mathrm{~min}\left(3.53 \log _{10} \mathrm{cfu} \cdot \mathrm{g}^{-1}\right)$ was the treatment which led to the best results for yeasts. However, we must bear in mind that with the stomacher method is not possible to distinguish between microorganisms which are only present in the superficial biofilms, or inside the fruits. In fact, Nychas et al. (2002) showed by SEM that a rich biofilm was developed on the epicuticular wax of the olive skin during fermentation, with yeasts dominated in the stomatal openings, but bacteria were more numerous in intercellular spaces in the sub-stomatal flesh. 

brines) and 11 yeast isolates ( 2 isolated from fruits and 9 from brines) were randomly obtained at the end of the fermentation process. A reduced number of yeast isolates was obtained because of the lower counts obtained from olive surface for this type of microorganisms at the end of fermentation.

The dendrogram generated by rep-PCR with primer GTG $_{5}$ using the patterns profile of the $20 \mathrm{LAB}$ isolates randomly obtained at the end of fermentation (Figure 5) showed that the isolates formed two groups clearly differentiated, sharing $78.8 \%$ similarity in their banding profile. The first group included isolates obtained from brines (7) and fruits (8), with a coefficient of similarity of $90.7 \%$. The second group presented a coefficient of similarity of $93.6 \%$, being formed by 2 isolates of fruits and 3 isolates of brine. Because of the reproducibility of the rep-PCR analysis for LAB was determined in $85.1 \%$, it was inferred that only two genotypes were present among the LAB population in the fermentation of natural green Gordal olives. Two representative isolates from each genotype (S5, S7, F10 and S9) were selected for identification purposes. Using the multiplex PCR method based on recA gene (Torriani et al. 2001) and RFLP analysis based on dnaK gene (Huang et al. 2010), all selected isolates were identified as Lactobacillus pentosus (multiplex PCR amplification of recA gene of $218 \mathrm{bp}$; profile of RFLP dnaK gene with TSP509I enzyme of $470+290+200+140 \mathrm{bp}$ ), thus indicating the presence of two different strains of the same species in the fermentation process. The presence of $L$. pentosus in vegetable fermentations, and particularly in biofilms of olives, has already been previously described (Arroyo-López et al. 2012a; Domínguez-Manzano et al. 2012; Grounta and Panagou 2014). 
of eleven yeast isolates, randomly selected from brines (9) or fruits (2) (Figure 6) showed the presence of two major groups sharing a low homology among them according to their banding profiles $(9.6 \%)$. Taking into account the technique reproducibility for yeasts (80.5\%), four different genotypes were distinguished. One representative isolate from each group (F2, S8, S3 and S4) was selected for identification purposes.

The restriction profiles generated by a battery of endonucleases on the 5.8-ITS region (Table 3) and further comparison in yeast data base, showed that isolates S4 and S8 obtained from brines belong to the same species ( $P$. galeiformis), while the isolate F2 obtained from fruits was identified as P. membranifaciens. Both yeast species have previously been isolated from diverse table olive elaborations (Arroyo-López et al. 2012b) and biofilms (Grounta and Panagou 2014). The profile restriction obtained for S3 isolate has not been found in the yeast database or in the literature, and further studies must be performed for its identification.

\section{Conclusions}

In the present study, it has been shown for the first time the formation of polymicrobial biofilms on natural green Gordal olives. The highest recovery of LAB from these biofilms was achieved by using the stomacher for $1 \mathrm{~min}$, while the highest yeast detachment was observed after sonication for 5 min. Thus, a combined treatment consisting of sonication and subsequent physical disaggregation of olives with stomacher could be very useful for a complete release of the different group of microorganisms, which should be confirmed in further studies. L. pentosus and P. membranifaciens were recovered from these biofilms at the end of the fermentation, with higher counts obtained for the 
bacteria. Hence, the study of the microorganisms forming biofilms on the epidermis of natural green table olives and the searching of those with beneficial properties is an interesting challenge because these fruits can also carry a high number of microorganisms $\left(>6.5 \log _{10} \mathrm{cfu} \cdot \mathrm{g}^{-1}\right)$. The use of natural olives for the development of potential probiotic olives is interesting due to is friendly (absence of lye treatment) and low energy cost processing.

\section{Acknowledgements}

The research leading to these results has received funding from the Spanish Government (project OliFilm AGL-2013-48300-R: www.olifilm.science.com.es), and the Junta de Andalucía (through financial support to group AGR-125). A. Benítez and F.N. Arroyo-López wish to express thanks to the Spanish Government for their pre-doctoral fellowship and postdoctoral research contract (Ramón y Cajal), respectively. V. Romero-Gil would like also to thank the AgriFood Campus of International Excellence (ceiA3), Bank of Santander, Spanish Government and 'Aloreña de Málaga' Olive Manufacturing Association for her pre-doctoral fellowship (training program of Ph.D. in companies).

\section{Conflict of interest}

The authors declare that they have not conflict of interest.

\section{References}

Aponte M, Ventorino V, Blaiotta G, Volpe G, Farina V, Avellone G, Lanza CM, Moschetti G (2010) Study of green Sicilian table olive fermentations through microbiological, chemical and sensory analyses. Food Microbiol 27:162-170. 
Arroyo-López FN, Bautista-Gallego J, Domínguez-Manzano J, Romero-Gil V, RodriguezGómez F, García-García P, Garrido-Fernández A, Jiménez-Díaz R (2012a) Formation of lactic acid bacteria-yeasts communities on the olive surface during Spanish-style Manzanilla fermentations. Food Microbiol 32:295-301.

Arroyo-López FN, Romero-Gil V, Bautista-Gallego J, Rodríguez-Gómez F, Jiménez-Díaz R, García-García P, Querol A, Garrido-Fernández A (2012b) Yeasts in table olive processing: desirable or spoilage microorganisms?. Int J Food Microbiol 160:42-49.

Balatsouras G (1990) Edible olive cultivars, chemical composition of fruit, harvesting, transportation, processing, sorting and packaging, styles of black olives, deterioration, quality standards, chemical analysis, nutritional and biological value of the end product. Olio d'oliva e olive da tavola: tecnologia e qualità 25:291-330.

Böckelmann U, Szewzyk U, Grohmann E (2003) A new enzymatic method for the detachment of particle associated soil bacteria. J Microbiol Methods 55:201-211.

Brenes M (2004) Olive Fermentation and Processing: Scientific and Technological Challenges. J Food Sci 69:FMS33-FMS34.

Brizuela MA, Serrano P, Pérez Y (2001) Studies on probiotics properties of two lactobacillus strains. Brazilian Arch Biol Technol 44:95-99.

Domínguez-Manzano J, Olmo-Ruiz C, Bautista-Gallego J, Arroyo-López FN, GarridoFernández A, Jiménez-Díaz R (2012) Biofilm formation on abiotic and biotic surfaces during Spanish style green table olive fermentation. Int J Food Microbiol 157:230-238. 
Esteve-Zarzoso B, Belloch C, Uruburu F, Querol A (1999) Identification of yeasts by RFLP analysis of the 5.8S rRNA gene and the two ribosomal internal transcribed spacers. Int J Syst Bacteriol 49:329-337.

Garrido Fernández A, Fernandez-Diez M, Adams MR (1997) Table olives: production and processing. Chapman and Hall, London (U.K).

Gevers D, Huys G, Swings J (2001) Applicability of rep-PCR fingerprinting for identification of Lactobacillus species. FEMS Microbiol Lett 205:31-36.

Grounta A, Panagou EZ (2014) Mono and dual species biofilm formation between Lactobacillus pentosus and Pichia membranifaciens on the surface of black olives under different sterile brine conditions. Annals Microbiol 64:1757-1767.

Huang C, Lee F, Liou J (2010) Rapid discrimination and classification of the Lactobacillus plantarum group based on a partial dnaK sequence and DNA fingerprinting techniques. Antonie van Leeuwenhoek 97:289-296.

Hurtado A, Reguant C, Bordons A, Rozès N (2012) Lactic acid bacteria from fermented table olives. Food Microbiol 31:1-8.

IOC (International Olive Oil Council) (2004) Trade standard applying to table olives. Resolution RES-2/91-IV/04. Madrid, Spain.

Kroupitski Y, Golberg D, Belausov E, Pinto R, Swartzberg D, Granot D, Sela S (2009) Internalization of Salmonella enterica in leaves is induced by light and involves chemotaxis and penetration through open stomata. Appl Environ Microbiol 75:60766086. 
Mason TJ, Paniwnyk L, Lorimer JP (1996) The uses of ultrasound in food technology. Ultrason Sonochem 3:S253-S260.

Medina E, Romero C, Brenes M, De Castro A (2007) Antimicrobial activity of olive oil, vinegar, and various beverages against foodborne pathogens. J Food Prot 70:11941199.

Nagpal R, Kaur A (2011) Synbiotic effect of various prebiotics on in vitro activities of probiotic lactobacilli. Ecol Food Nutr 50:63-68.

Nychas GE, Panagou EZ, Parker ML, Waldron KW, Tassou CC (2002) Microbial colonization of naturally black olives during fermentation and associated biochemical activities in the cover brine. Lett Appl Microbiol 34:173-177.

Peréz-Díaz IM, Breidt F, Buescher RW, Arroyo-López FN, Jiménez-Díaz R, GarridoFernández A, Bautista-Gallego J, Yoon SS, Johanningsmeire SD (2013) Fermented and Acidified Vegetables. In: Compendium of methods for the microbiological examination of foods. $4^{\text {th }}$ Edition (Ed. Pouch-Downes, F., and Ito, K.). American Public Health Association, Washington, USA. pp- 521-532.

Perricone M, Bevilacqua A, Corbo MR, Sinigaglia M (2010) Use of Lactobacillus plantarum and glucose to control the fermentation of "Bella di Cerignola" table olives, a traditional variety of Apulian region (southern Italy). J Food Sci 75:M430-M436.

Piyasena P, Mohareb E, McKellar R (2003) Inactivation of microbes using ultrasound: a review. Int J Food Microbiol 87:207-216.

Raffin J, Peyron I, Sarfati A (2008) Ultrasounds in sterilization: Study on recommendations follow-up. Pharmacien Hospitalier 43:81-86. 
Sala Trepat FJ (1995) Food preservation through combined processes. Microbiol SEM 11:23-32.

Sánchez AH, De Castro A, Rejano L, Montaño A (2000) Comparative study on chemical changes in olive juice and brine during green olive fermentation. J Agric Food Chem 48:5975-5980.

Tassou CC, Panagou EZ, Katsaboxakis KZ (2002) Microbiological and physicochemical changes of naturally black olives fermented at different temperatures and $\mathrm{NaCl}$ levels in the brines. Food Microbiol 19:605-615.

Tofalo R, Chaves-López C, Di Fabio F, Schirone M, Felis GE, Torriani S, Paparella A, Suzzi G (2009) Molecular identification and osmotolerant profile of wine yeasts that ferment a high sugar grape must. Int J Food Microbiol 130:179-187.

Torriani S, Felis GE, Dellaglio F (2001) Differentiation of Lactobacillus plantarum, L. pentosus, and L. paraplantarum by recA Gene Sequence Analysis and Multiplex PCR Assay with recA Gene-Derived Primers. Appl Environ Microbiol 67:3450-3454.

Tsukamoto I, Yim B, Stavarache CE, Furuta M, Hashiba K, Maeda Y (2004) Inactivation of Saccharomyces cerevisiae by ultrasonic irradiation. Ultrason Sonochem 11:61-65.

Willey JM, Sherwood L, Woolverton CJ (2011) Prescott's microbiology, McGraw-Hill.

Zwietering MH, Jongenburger I, Rombouts FM, Van't Riet K (1990) Modeling of the bacterial growth curve. Appl Environ Microbiol 56:1875-1881. 
493

494

495

496

497

498

499

500

501

502

503

504

505

506

507

508

509

510

511

512

513

514

515

516

517

518

Figure 1. Evolution of $\mathrm{pH}(\mathrm{a})$ and titratable acidity (b) throughout fermentation of Gordal directly brine natural table olives.

Figure 2. Reparameterized Gompertz equation fit to the plate counts $\left(\log _{10} \mathrm{cfu} \cdot \mathrm{ml}^{-1}\right)$ of $\mathrm{LAB}$ (a) and yeast (b) populations in brines throughout the fermentation process of directly brined Gordal variety olives.

Figure 3. SEM pictures obtained from the epidermis of natural green Gordal olives after 90 days of fermentation. Arrows indicate LAB and yeasts surrounded by a matrix in the biofilms.

Figure 4. Counts $\left(\log _{10} \mathrm{cfu} \cdot \mathrm{g}^{-1}\right)$ of the LAB and yeasts populations obtained after application of different sonication times, enzymatic concentrations, stomacher and ultrasonic bath times to the biofilms formed on the skin of directly brined Gordal olives. The means and the associated deviations were obtained from $n=6$ measurements for each level. Temperature in the ultrasonic bath was kept constant at $30^{\circ} \mathrm{C}$ by ice addition.

Figure 5. Dendrogram generated after bioinformatic analysis with Bionumerics 6.6 software package of the rep-PCR profiles obtained with $\mathrm{GTG}_{5}$ primer for the different LAB randomly isolated from brines (S) or biofilms (F) at the end of fermentation ( $90 \mathrm{~d}$ ).

Figure 6. Dendrogram generated after bioinformatic analysis with Bionumerics 6.6 software package of the RAPD-PCR profiles obtained with M13 primer for the different yeast isolates randomly obtained from brines $(\mathrm{S})$ or biofilms $(\mathrm{F})$ at the end of fermentation (90 d). 
Table 1. Average plate counts $\left(\log _{10} \mathrm{cfu} \cdot \mathrm{g}^{-1}\right)(\mathrm{n}=6)$ of the LAB and yeasts populations adhered to the olive surface after application of the different detachment methods and levels.

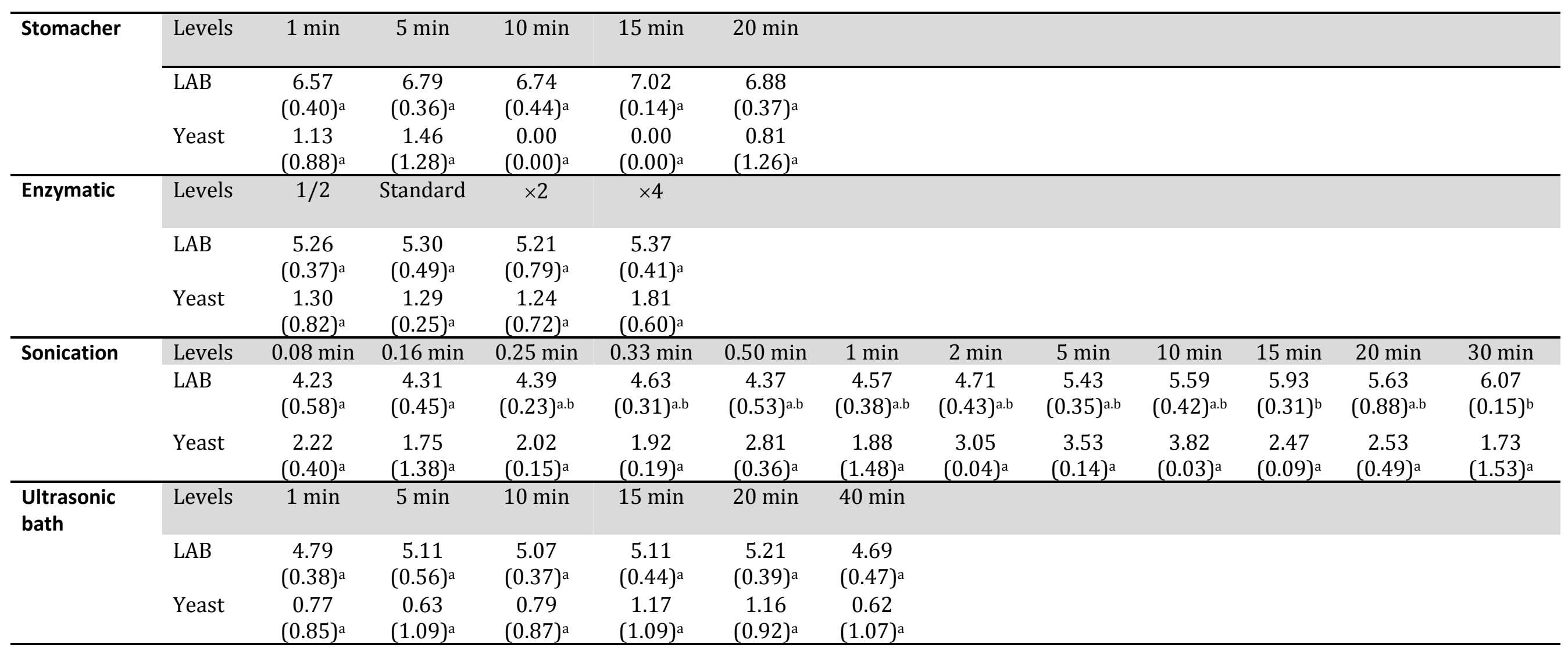

Note: Standard deviation in parentheses. Values followed by different superscript letters, within the same row, are significantly different according to Scheffé post-hoc comparison test. 
Table 2. One-way ANOVA analysis for the comparison among the best levels of the diverse detachment methods for lactic LAB and yeasts populations.

\begin{tabular}{lcc}
\hline Procedure $/$ Level & LAB $\left(\log _{10} \mathrm{cfu} \cdot \mathrm{g}^{-1}\right)$ & Yeast $\left(\log _{10} \mathrm{cfu} \cdot \mathrm{g}^{-1}\right)$ \\
\hline Stomacher $(1 \mathrm{~min})$ & $6.57(0.40)^{\mathrm{a}}$ & $1.13(0.88)^{\mathrm{a}}$ \\
Enzymatic $(1 / 2)$ & $5.25(0.37)^{\mathrm{b}}$ & $1.30(0.82)^{\mathrm{a}}$ \\
Sonication $(5 \mathrm{~min})$ & $5.43(0.35)^{\mathrm{b}}$ & $3.53(0.14)^{\mathrm{b}}$ \\
Ultrasonic bath $(1 \mathrm{~min})$ & $4.79(0.38)^{\mathrm{b}}$ & $0.77(0.85)^{\mathrm{a}}$ \\
\hline
\end{tabular}

Note: Standard deviations are in parentheses. Values followed by different superscript letters, within the same column, are significantly different according to Scheffé post-hoc comparison test. 
Table 3. RFLP profiles (in bp) for the 5.8-ITS region of the four selected yeast isolates from rep-PCR analysis with M13 primer.

\section{Restriction enzyme}

\begin{tabular}{llllll}
\hline Isolates & PCR & CfoI & HaeIII & Hinfl & Species \\
\hline S-3 & 480 & $270+250+100+70$ & $320+90+50$ & $300+250+200+190$ & Unknown profile \\
S-4 & 460 & $250+100+60$ & $320+90+50$ & $250+200$ & Pichia galeiformis \\
S-8 & 460 & $250+100+60$ & $320+90+50$ & $250+200$ & Pichia galeiformis \\
F-2 & 490 & $190+110+90$ & $320+90+50$ & $275+200$ & Pichia membranifaciens
\end{tabular}

519 
Figure 1
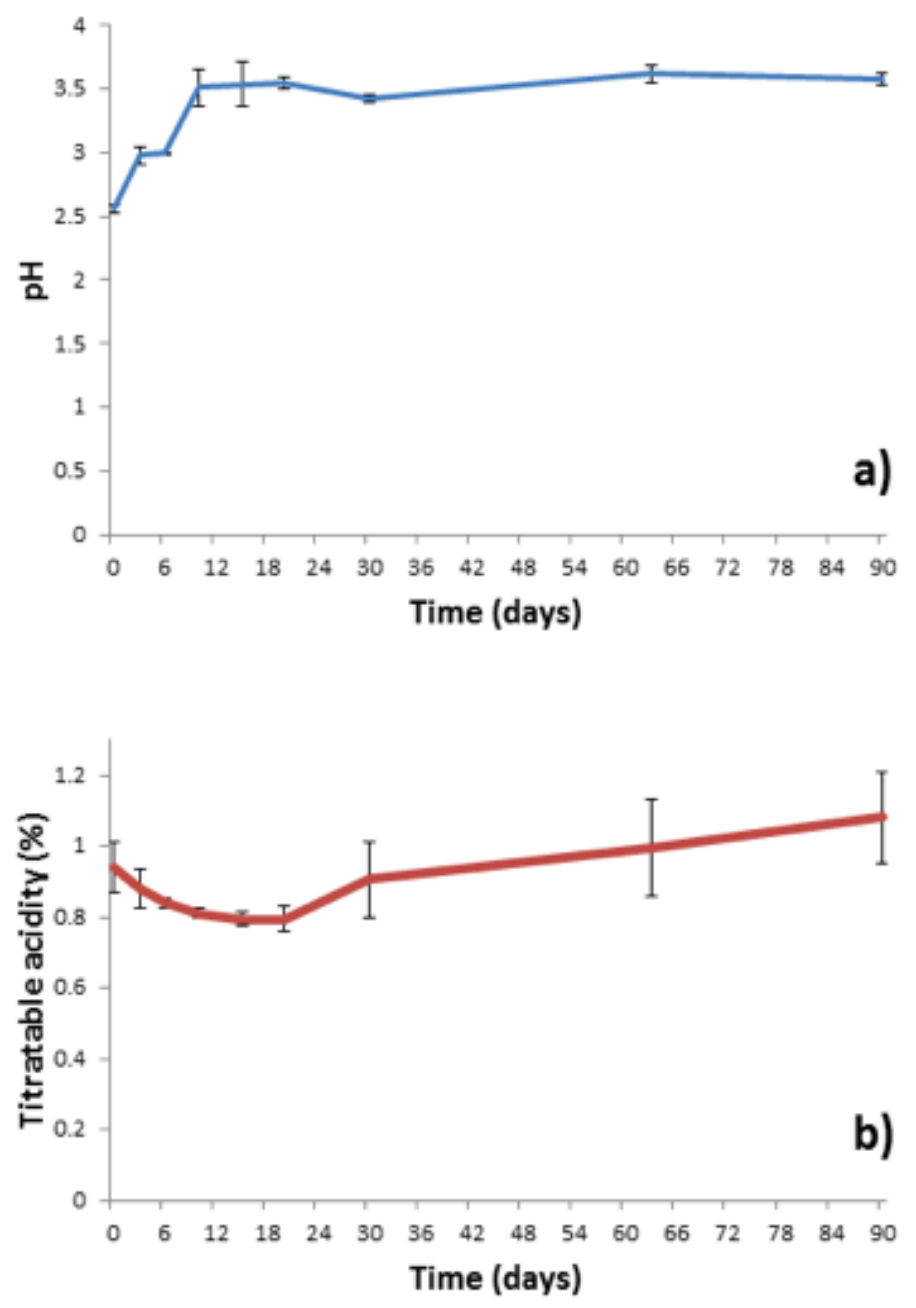
Figure 2
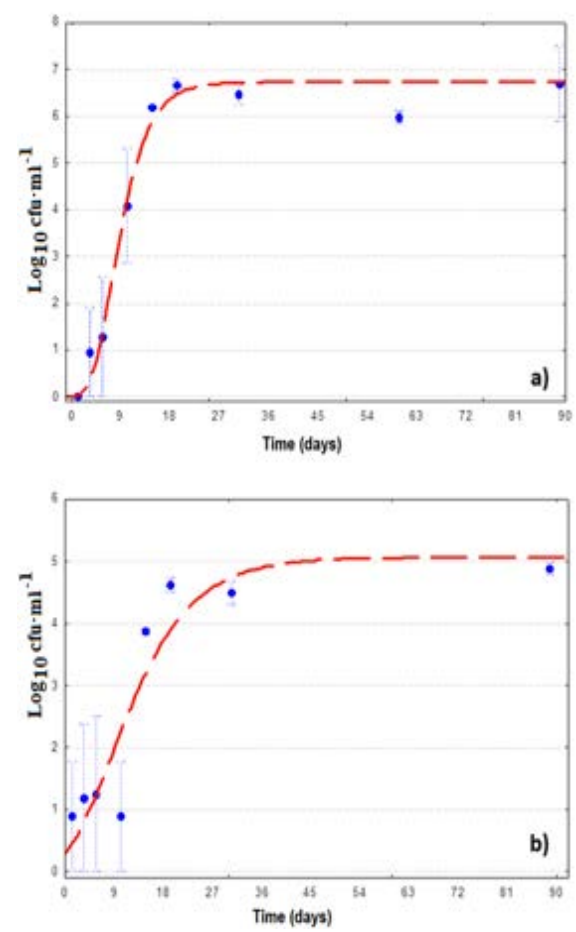

521 
Figure 3
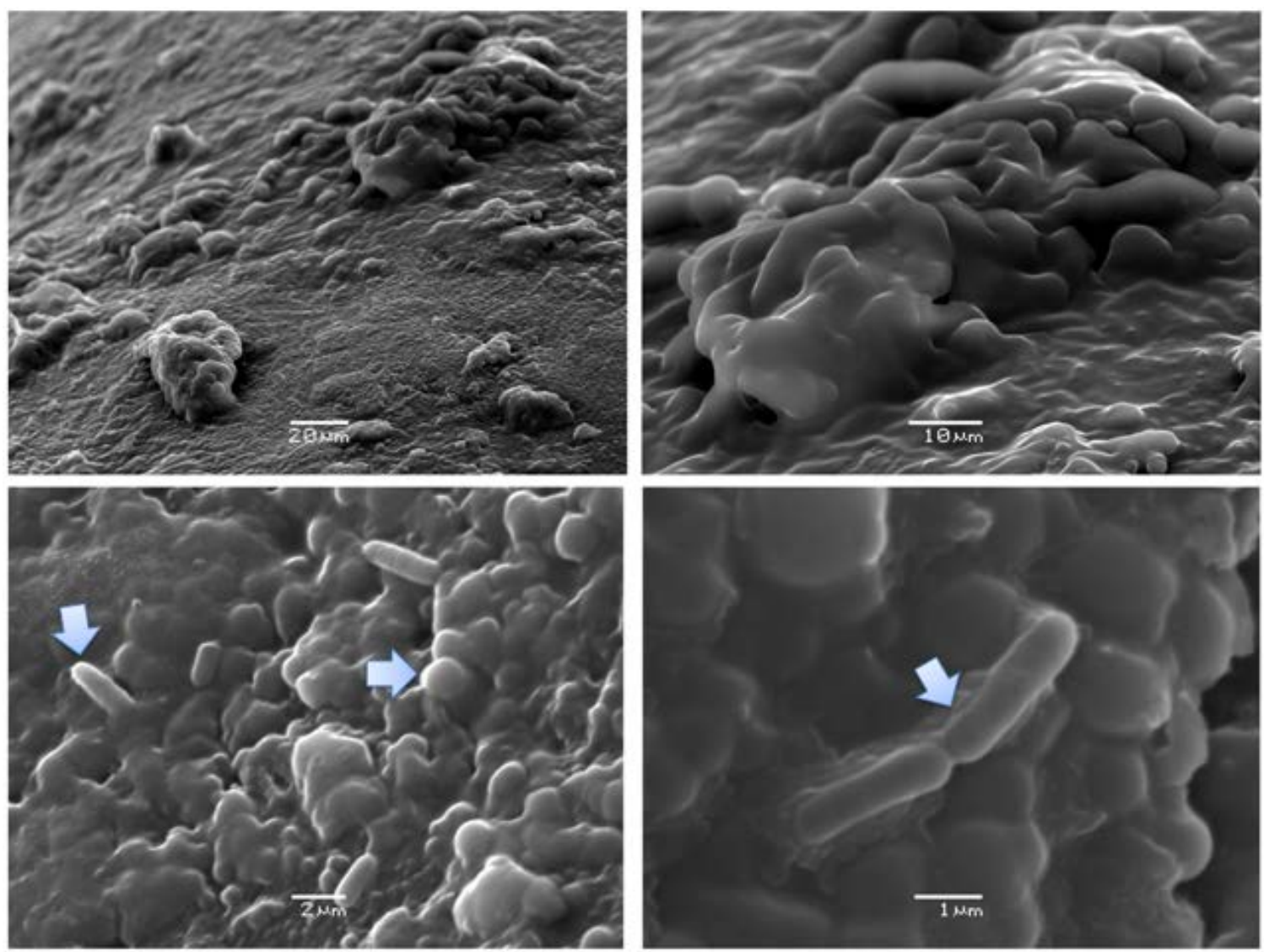

522 
Figure 4

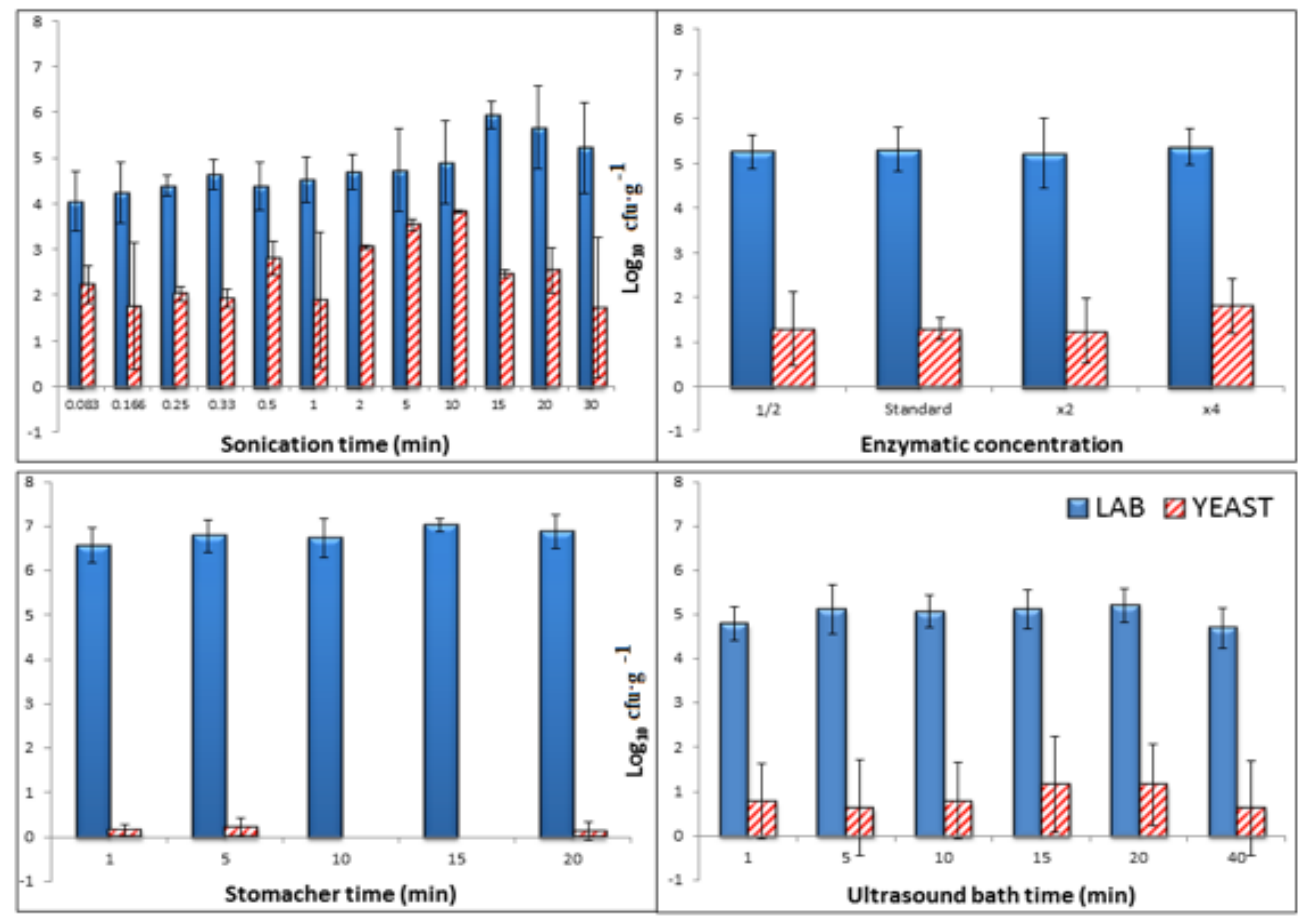


Figure 5

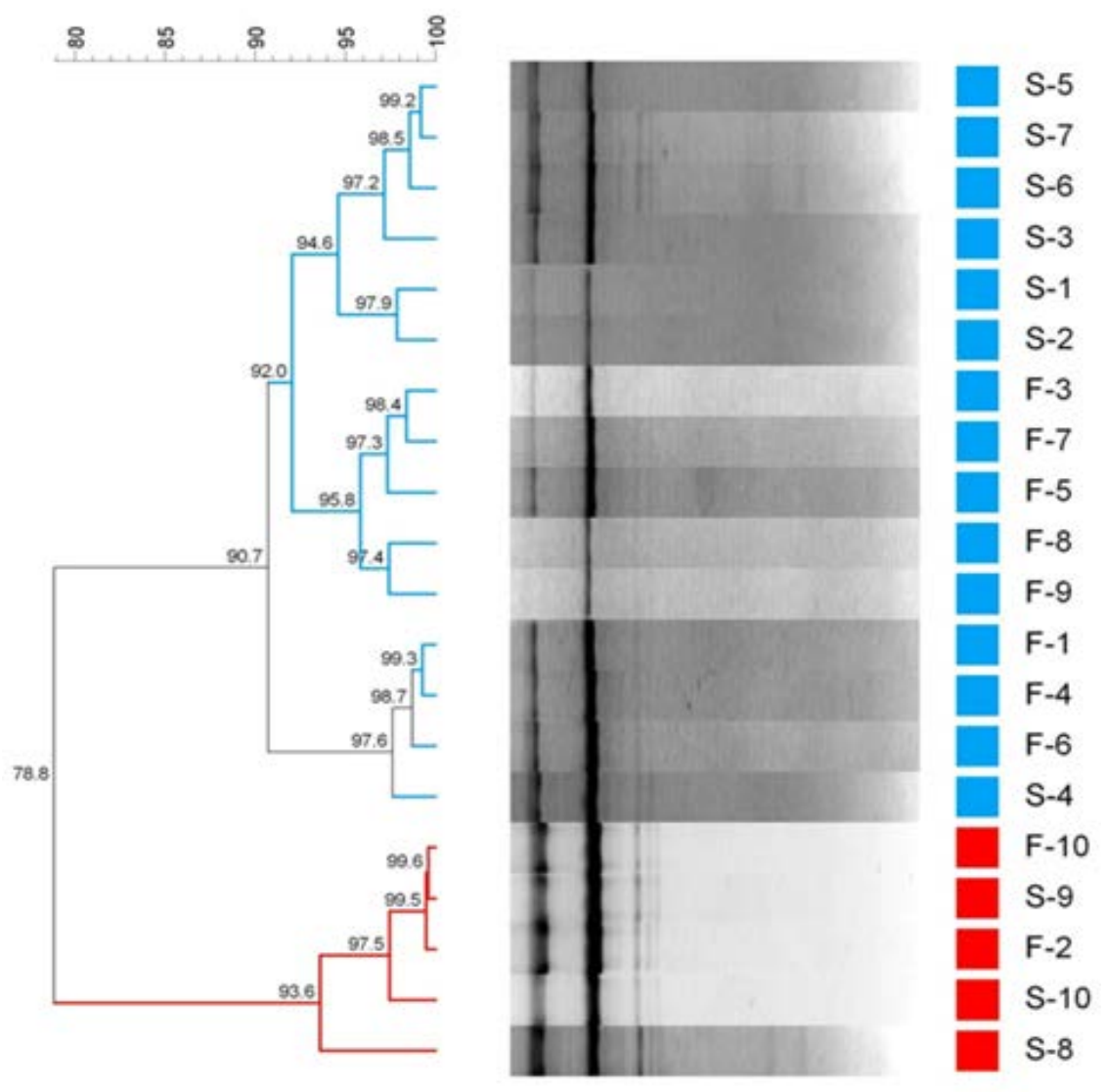


Figure 6

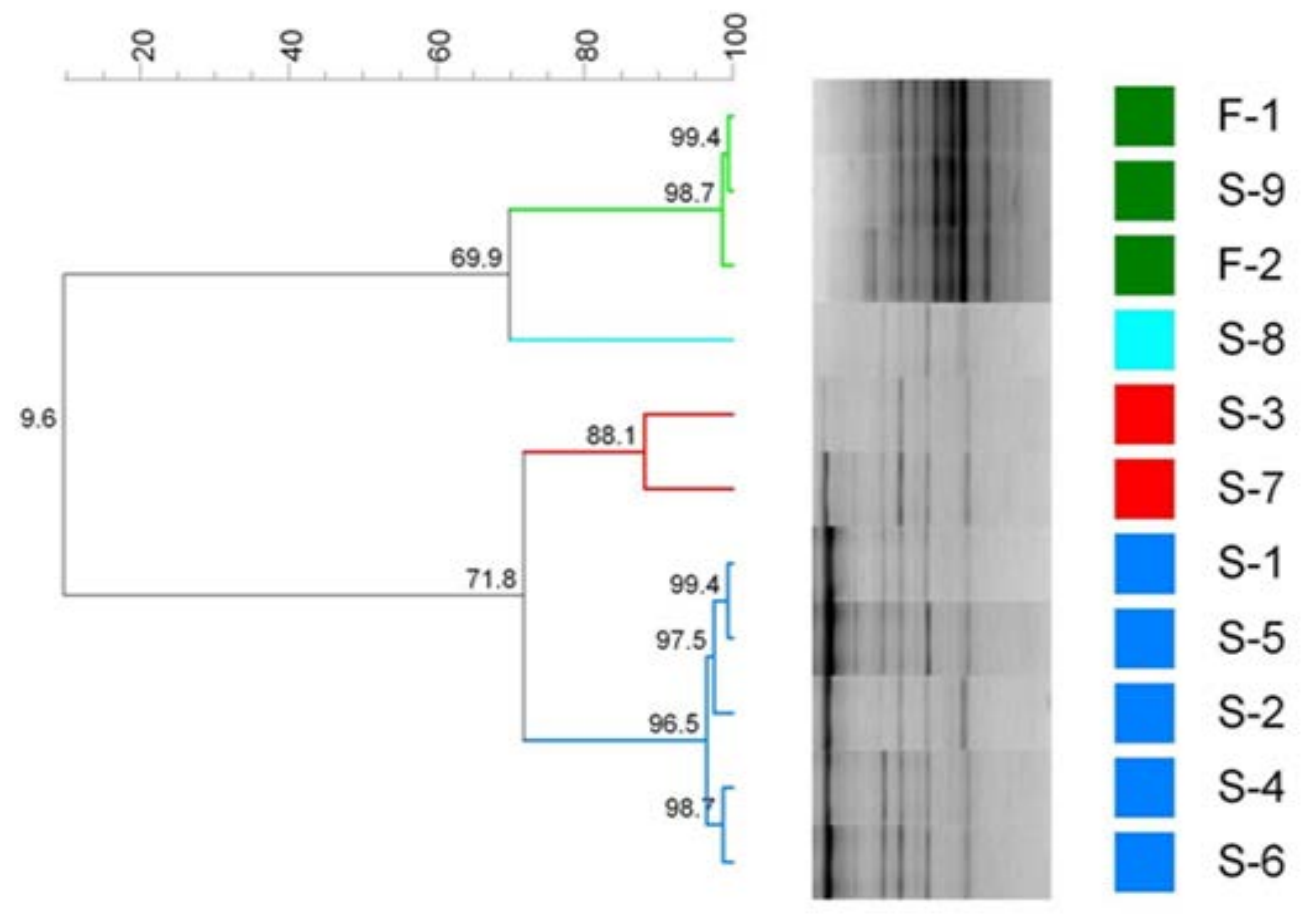

